\title{
Design of Broadband non-Foster Circuits based on Resonant Tunneling Diodes
}

\author{
Deepak Singh Nagarkoti, Student Member, IEEE, Yang Hao, Fellow, IEEE, David P. Steenson, Lianhe Li, \\ Edmund H. Linfield and Khalid Z. Rajab, Member, IEEE
}

\begin{abstract}
A non-Foster circuit (NFC) based on the resonant tunneling diode (RTD) is proposed for application to broadband impedance matching of electrically small antennas (ESAs). NFCs have traditionally been implemented with transistor pairs to achieve negative impedance, but these have limitations with respect to performance and operational bandwidth at high frequencies. At certain biasing voltages, double barrier RTDs behave as negative differential resistance (NDR) devices, which may be transformed to exhibit negative impedance. In contrast to the transistor-based NFC, these structures serve to gyrate or invert the load impedance, such that an inductive load will lead to a negative capacitance, and vice versa. This device is termed a negative impedance inverter (NII). We demonstrate negative impedance behavior for prototypes with measurements of negative resistance at up to $3 \mathrm{GHz}$, and device gain of around $5 \mathrm{~dB}$ from DC to $4 \mathrm{GHz}$. Design for stability of the RTD is performed using the Nyquist stability criterion. Stabilized negative capacitance NFCs show optimum performance from DC to the GHz range depending upon the load value. These NFCs are used to impedance match an antenna at low frequencies. An antenna with only one resonance at $3.5 \mathrm{GHz}$ has been transformed with two different matching circuits: to an antenna encompassing the 1 to $2 \mathrm{GHz}$ range; as well as the VHF/UHF bands from $300 \mathrm{MHz}$ to $1 \mathrm{GHz}$. Additionally, RTDs have been demonstrated for operation at up to $\mathrm{THz}$ frequencies, so this topology can be extended to higher frequencies subject to fabrication constraints.
\end{abstract}

Index Terms-resonant tunneling diodes (RTDs), non-Foster circuits (NFCs), stability, electrically small antenna (ESA).

\section{INTRODUCTION}

Likely the most well-known negative impedance converter (NIC) topology is based on a design by Linvill [1], in which a bipolar junction transistor (BJT) pair flips the direction of the current (or voltage) through a load, whilst maintaining the polarity of the voltage (or current). Circuit implementations are widely referred to as non-Foster circuits (NFCs) as the negative slope of the reactance is contrary to Foster's theorem for lossless, passive devices, which must always show a positive slope for reactance with frequency. Printed NFCs have been investigated and developed for a number of applications, such as broadband impedance matching of small antenna in order to exceed the Bode-Fano matching

Deepak Singh Nagarkoti, Yang Hao and Khalid Z. Rajab are with the Antenna and Electromagnetics Research Group, Queen Mary University of London, London, E1 4NS, United Kingdom (e-mail: k.rajab@qmul.ac.uk). David P. Steenson, Lianhe Li and Edmund H. Linfield are with the Institute of Microwaves and Photonics, School of Electronic and Electrical Engineering, University of Leeds, Leeds LS2 9JT, United Kingdom (e-mail: e.h.linfield@leeds.ac.uk).

Manuscript received May 28, 2015

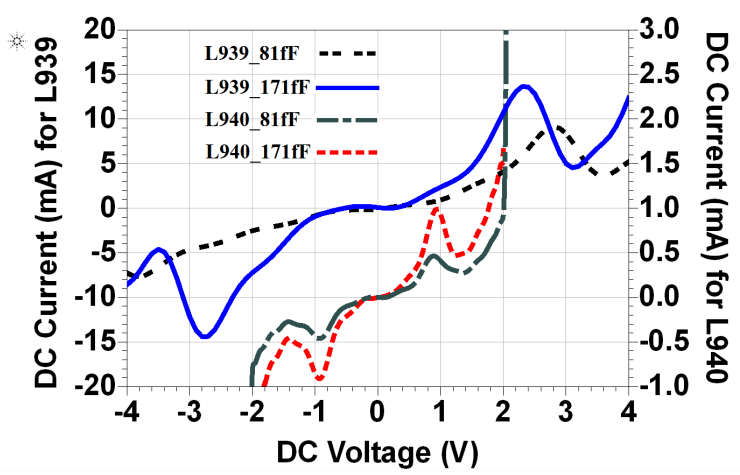

Fig. 1. Measured I-V characteristics of the four RTD samples. Negative differential resistance regions are present at $\sim \pm 3 \mathrm{~V}$ for the L939 samples, and at $\sim \pm 1 \mathrm{~V}$ for the L940 samples. The best sample L939 with $171 \mathrm{fF}$ capacitance has been used with biasing at $2.75 \mathrm{~V}$.

limitations [2], [3] which states that it is in general difficult to impedance match electrically small antennas (ESAs) due to their high $Q$ [4]. Additionally, there has recently been interest in the application of NFCs for improving the bandwidth of metamaterial structures [5], [6], [7]. Due to size constraints and design complexity, it is difficult to incorporate these BJT based NFCs within each element of a periodic metamaterial structure. Alternatives to the Linvill NFC approach have been implemented through SiGe BiCMOS processes to overcome size limitations, reduce parasitics, and extend operational frequencies [8], [9].

In this letter we demonstrate a method for implementing a non-Foster device based on the resonant tunneling diode (RTD). The RTD-based device behaves as a negative impedance inverter (NII) by creating an effective negative inductance from a capacitive load, or a negative capacitance from an inductive load. In section II we describe the operation, modeling, and fabrication of the RTD. Stability of any active device is an important design consideration, and in section III we analyze the NFC stability. Section IV is dedicated to the implementation of various NFCs using RTDs. Finally, in section $\mathrm{V}$ we demonstrate a method for impedance matching of a small antenna using the RTD-based NFC.

\section{Modeling AND FABrication OF THE RTD}

A resonant tunneling diode is so because the transmission of electrons, and therefore current, depends on the alignment between the filled electron states in the emitter with the resonant quasi-confined allowed states in the well. This results in negative differential resistance and a negative slope in the 
current versus voltage characteristic when the structure is biased just beyond resonance. This region of biasing where the RTD operates as a negative resistance device is used for the NFC design proposed here, and will be utilized as a basic element to realize floating and grounded negative reactances.

RTDs exhibit voltage-controlled behavior in that a singlevalued current is obtained at different values of voltage. The high frequency operational limit of an RTD is defined by the charging time constant of the device capacitance. Hence, low capacitances equate to high speed devices. Consideration of junction capacitance, negative resistance, parasitic resistance and load coupling is required to model RTDs accurately at microwave frequencies [10].

RTD samples were fabricated using GaAs technology. Two materials were used with barrier thicknesses of $1.7 \mathrm{~nm}$ AlAs (L939) and $5 \mathrm{~nm}$ thick $\mathrm{Al}_{(0,4)} \mathrm{Ga}_{(0,6)} \mathrm{As}(\mathrm{L} 940)$ with with similar well thicknesses of $5 \mathrm{~nm}$. There two different top contact lengths for each material, creating four separate samples, two with a capacitance of $81 \mathrm{fF}$ and two with $171 \mathrm{fF}$. The devices were mounted on high frequency lead-less inductive device packages and wire bonded, ready for use as surfacemount devices. The I-V characteristics were measured using a Cascade Microtech probe station and LCR meter. Each set of measurement data was modeled to a polynomial function using a curve fitting tool, and then exported to a circuit simulator. Measurement data for these samples are plotted in Fig. 1, with negative differential resistance (NDR) regions clearly visible. As discussed before, the RTD must be biased at the voltage level corresponding to the negative slope. NDR regions with sharp transitions are generally not preferred for selection of the biasing point, as they lead to high current sensitivity with voltage variation. The L939 sample with $171 \mathrm{fF}$ capacitance shows the most suitable negative resistance region for our purpose.

All four samples have also undergone RF characterization, with measured $S_{21}$ plotted in Fig. 2. The L939 sample with $171 \mathrm{fF}$ capacitance shows maximum transmission gain of around $5 \mathrm{~dB}$ up to $4 \mathrm{GHz}$. The RF performance of the diode has been modeled as a two port network and the impedance parameters have been extracted for different biasing points as shown in Fig. 3. For a biasing level of $2.75 \mathrm{~V}$, the resistance measured in the device is around $-50 \Omega$ at low frequencies, and increases with frequency. Any additional reactance offered by the device is small as compared to the lower frequency resistance. It shows the expected effective contribution beyond $3 \mathrm{GHz}$, which contributes significantly to degrade the expected circuit performance beyond this point.

\section{STABILITy ANALYSIS OF THE RTD}

Due to the operation of the diode in the negative resistance mode, there are concerns with possible oscillations and instability. The circuit model analysis for the stable operation of the RTD has been investigated using the differential equations method [11]. Later, Frisch came up with a new sufficient condition wherein all poles in a linear equivalent circuit of the diode shown in [12] can be shifted to the left half of the complex $s$-plane for the diode to be considered potentially

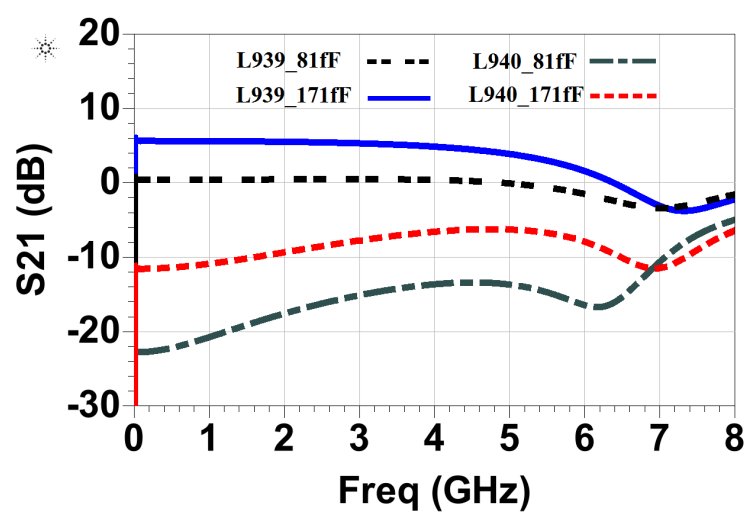

Fig. 2. Transmission gain of different RTD samples biased at their individual negative resistance region. Sample L939 with $171 \mathrm{fF}$ capacitance exhibits $5 \mathrm{~dB}$ gain up to $4 \mathrm{GHz}$ and positive gain at up to around $6 \mathrm{GHz}$, however this may lead to instability and oscillation of the device.

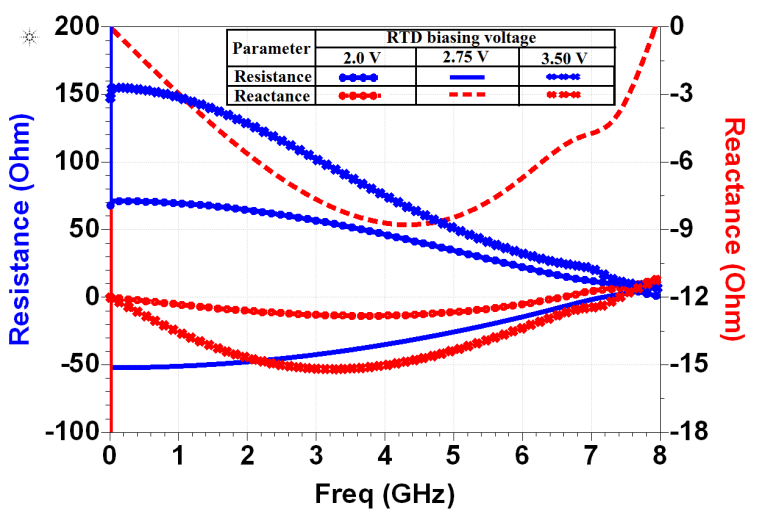

Fig. 3. RF resistance and reactance shown by the RTD sample L939 with $171 \mathrm{fF}$ capacitance, biased in the different voltages around the negative resistance region. The negative resistance and minimum reactance is noted at $2.75 \mathrm{~V}$.

stable. Recent work by Stearns [13], [14] verifies that polezero analysis is not suitable for high-order complex network and that the $\mu$-test for unconditional stability [15] is not suitable for NFCs. The frequency domain Nyquist criterion [16] consider the natural frequencies of the complete circuit. Fig. 4 shows that the Nyquist contour no longer encircles the origin point as the series resistor increases from $50 \Omega$ to $55 \Omega$, and hence selecting the latter will avoid oscillatory behavior.

\section{Design Methodology of NFCs}

An NFC in general is an active n-port network designed in violation of Foster reactance theorem in which the reactance or susceptance decreases with increasing frequency. The one port short-circuited NFC has non-Foster behavior at the input port. Similarly, the two port floating NFC exhibits this property across the ports. Transistor-based NFCs have been explored recently for active matching of ESAs [17]. The stable negative resistance RTD element discussed in previous sections is used to develop a bilateral design for negative capacitance and inductance. A unique feature in these non-Foster designs is that all components except the RTD element are passive RLC components. Thus, it highly reduces the complexity of the circuit realization once a potentially stable RTD is 


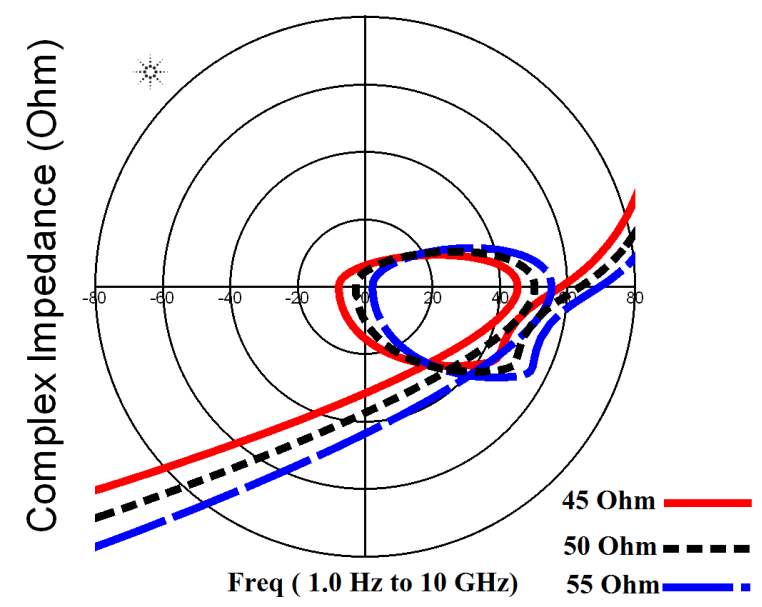

Fig. 4. Test of RTD stability using the Nyquist stability criterion, for determination of stable component values. Encirclement of the origin in the complex plane implies instability.

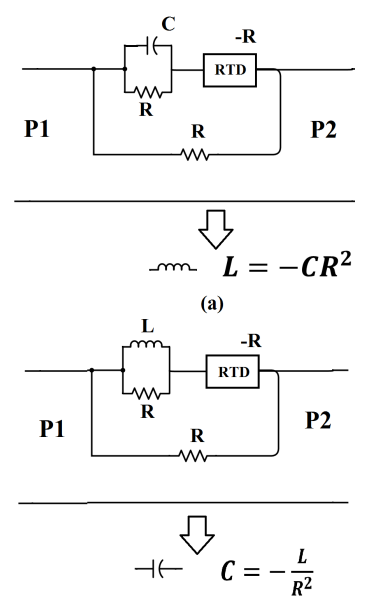

(c)

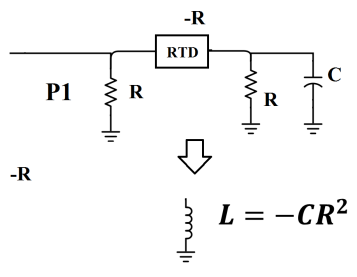

(b) $-\mathrm{R}$

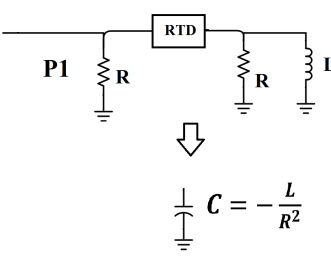

(d)
Fig. 5. Design topologies of various NFCs using RTD elements. (a) A series negative inductor and (b) a shunt negative inductor made of an RTD, resistors and a capacitor. (c) A series negative capacitor and (d) a shunt negative capacitor made of an RTD, resistors and an inductor.

obtained. Schemes for designing various open-circuited and short-circuited, inductive and capacitive NFCs are shown in Fig. 6. The two port open-circuited design approach has been proposed here, while a one port short-circuited version was presented in [18]. In each proposed design, both resistor elements $R$ provide the required stability for smooth RTD operation and furthermore, the stability of the NFC. This has been verified with the Nyquist stability criterion discussed in the previous section. It is further noted that an inductive load results in a negative capacitance NFC, while a capacitive load gives a negative inductance NFC. In effect, this property of inverting the current-voltage characteristics of a network results in a negative gyrator or NII. This may find application at high frequencies, where implementation of negative inductors with inductive loads is troublesome.

Another property is that ideally, the resistor value is equal to the magnitude of the negative resistance offered by the RTD element. As the RTD has frequency dependent resistance, the

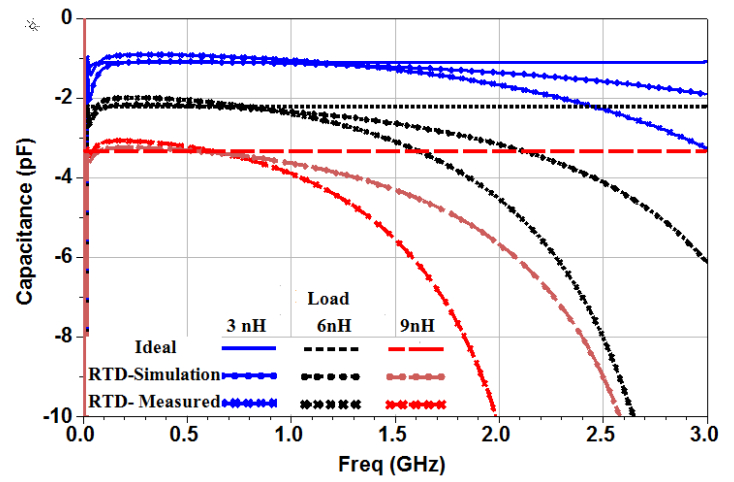

Fig. 6. Negative capacitor NFC following the circuits from Figs. 5(c) or (d). The figure shows the effect of negative capacitance due to variation of load inductance values, and comparisons between using an ideal, simulated and measured RTD model. In either case, negative capacitance may be achieved, but bandwidth is minimum for the measured scenario due to the effective dominance of parasitic reactance.

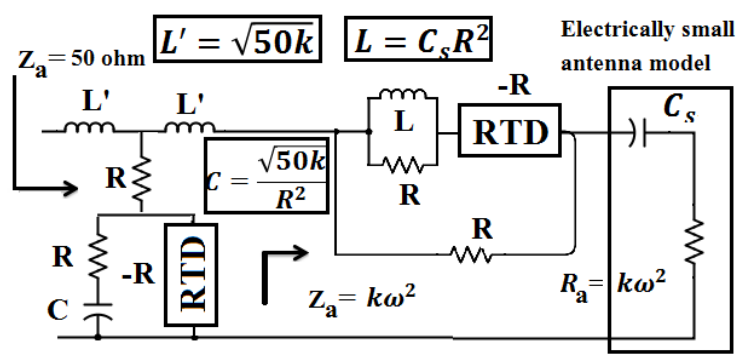

Fig. 7. Matching of a capacitive antenna with two RTD devices. The capacitive part has been matched using the floating NFC circuit of Fig. 5(c). The resistive matching involves an RTD transformed to $-k \omega^{2}$.

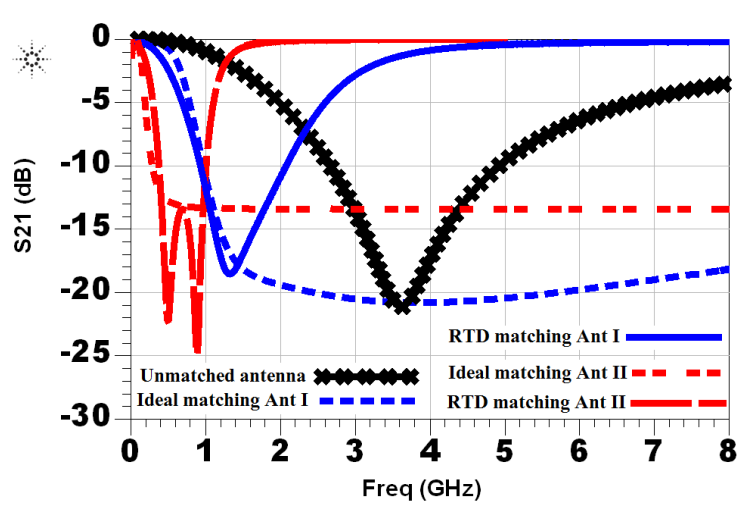

Fig. 8. Reflection coefficient comparison with different matching approaches. The unmatched antenna resonates at around $3.5 \mathrm{GHz}$, and shows very poor matching at low frequencies. By varying the matching circuit with the elements from Table I, matching is demonstrated below $1 \mathrm{GHz}$, and from 1 to $2 \mathrm{GHz}$.

negative inductance and capacitance obtained also vary with frequency. Fig. 7 shows the realization of negative capacitance using an inductive load, following the circuit from Fig. 5(c) or Fig. 5(d). The decreased bandwidth of the fabricated NFC as compared to the ideal case is evident. The RTD may similarly be used to synthesize a negative inductor. 
TABLE I

MATCHING ELEMENTS FOR ANTENNA WITH 5 PF CAPACITANCE AND FREQUENCY-DEPENDENT RADIATION RESISTANCE $R_{a}=k \omega^{2}$. ANT I IS MATCHED IN THE 1 TO 2 GHZ BAND. ANT II IS MATCHED AT VHF/UHF.

\begin{tabular}{|c||c||c||c|}
\hline Circuit elements & Ideal matching & ANT I & ANT II \\
\hline Resistor (R) & -(RTD resistance) & $52.7 \Omega$ & $60.2 \Omega$ \\
\hline Inductor 1 (L) & $C_{s} R^{2}$ & $41 \mathrm{nH}$ & $6 \mathrm{nH}$ \\
\hline Inductor 2 (L') & $\sqrt{50 k}$ & $31 \mathrm{nH}$ & $13 \mathrm{nH}$ \\
\hline Capacitor (C) & $\frac{\sqrt{50 k}}{R^{2}}$ & $12.5 \mathrm{pF}$ & $1.6 \mathrm{pF}$ \\
\hline
\end{tabular}

\section{IMPEDANCE MATCHING OF AN ESA MODEL}

Due to the large reactive input impedance and very low radiation resistance of an ESA in VHF/UHF band, very little power is radiated to the far-field region and most of the input power is reflected or stored in the reactive near-field region. The quality factor $Q$ may be reduced towards Chu's limit [4], thus increasing bandwidth, through various schemes, such as the use of multiple matching stages. However there exists a fundamental gain-bandwidth restriction between the generator and load, and a finite bandwidth which can be achieved, even with a large number of matching section. Furthermore, circuit size and complexity will increase, and efficiency will go down as losses add up.

It is possible to overcome the gain-bandwidth limitations with a non-Foster approach using NFCs. The radiation resistance of an ESA has a frequency-squared dependence that is small at low frequencies (a few $\Omega$ ). The reactive and resistive matching of the ESA has been performed with the RTD-based NFCs, and is shown in Fig. 8. Two RTD NFC stages are required for matching: one each for resistive and reactive matching. An RTD-based capacitive NFC (nearest to the ESA) first cancels the positive antenna capacitance, leaving an approximately real impedance. The next stage is a resistive matching network that transforms the frequency dependent antenna resistance $R_{a}=k \omega^{2}$ to $50 \Omega$ (or some other constant characteristic line impedance).

The antenna is self-resonant at $3.5 \mathrm{GHz}$, which corresponds to a monopole of length approximately $50 \mathrm{~mm}$; this would be considered electrically small $(\lambda / 10)$ up to $600 \mathrm{MHz}$. It may be modeled up to first resonance as a capacitor $C=5 \mathrm{pF}$ and a resistor with parameter $k=10^{-19} \Omega \cdot s^{2}$. Using the measured RTD parameters, the antenna is matched through simulation for two cases, using the parameters from Table I in the circuit from Fig. 7. The improvement in impedance matching and bandwidth is evident from Fig. 8: ANT I is matched such that the lower frequency of the matched band has been reduced to $1 \mathrm{GHz}$; ANT II is matched to encompass the VHF and UHF bands, up to around $1 \mathrm{GHz}$. In both cases, measured parameters from the RTD is used in simulation of Fig. 8. We note that an ideal RTD with reduced junction capacitance would match the antenna from $150 \mathrm{MHz}$ to above $5 \mathrm{GHz}$.

\section{CONCLUSION}

A novel implementation of a NFC is demonstrated, which takes advantage of the NDR of RTDs. To create a negative impedance, it is shown that the device operates as a negative gyrator or NII, exhibiting stable negative reactance and resistance (i.e. gain) into the $\mathrm{GHz}$ regime, and differs significantly from previously demonstrated transistor-based devices. We also note that the bandwidth of these NFCs may be significantly improved through reduction of the device's junction capacitance. It is also shown that these NFC devices may be used to impedance match both the resistive and reactive parts of an ESA, over bandwidths that are not possible using passive matching techniques. An antenna that was selfresonant at $3.5 \mathrm{GHz}$ was matched to better than $10 \mathrm{~dB}$ return loss for two cases: from 1 to $2 \mathrm{GHz}$; and for VHF/UHF bands up to $1 \mathrm{GHz}$. This could be potentially useful for use in lowpower, wide-band receive applications.

\section{REFERENCES}

[1] J. Linvill, "Transistor negative impedance converters," Proc. IRE, vol. 41, pp. 725-729, June 1953.

[2] H.W. Bode, "Network Analysis and Feedback Amplifier Design," New York, NY, USA:, D. Van Nostrand, 1945.

[3] R.M. Fano, "Theoretical limitations on the broad-band matching of arbitrary impedances," J.Franklin Institute, vol. 249, pp. 57-83, Jan. 1950 and pp. 139-154, Feb. 1950.

[4] L. Chu, "Physical limitations of omnidirectional antennas," J. Appl. Phys., vol. 19, pp. 1163-1175, Dec 1948.

[5] S. A. Tretyakov, "Meta-materials with wideband negative permittivity and permeability," Microw. Opt. Techn. Lett., vol. 31, issue 3, pp. 163-165, 2001.

[6] J. Long, M. M. Jacob and D. F. Sievenpiper, "Broadband fast-wave propagation in a non-Foster circuit loaded waveguide," IEEE Trans. Microw. Theory Techn., vol. 62, no. 4, pp. 789-798, Apr. 2014.

[7] E. Ugarte-Munoz, S. Hrabar, D. Segovia-Vargas and A. Kiricenko, "Stability of non-Foster reactive elements for use in active metamaterials and antennas," IEEE Trans. Antennas Propag., vol. 60, no. 7, pp. 34903494, Jul. 2012.

[8] D. J. Gregoire, C. R. White and J. S. Colburn, "Wideband artificial magnetic conductors loaded With non-Foster negative inductors," IEEE Antennas Wireless Propag. Lett., vol. 10, pp. 1586-1589, Dec. 2011.

[9] S. Saadat, H. Aghas, E. Afshari and H. Mosallaei, "Low-power negative inductance integrated circuits for GHz applications," IEEE Microw. Compon. Lett., vol. 99, pp. 118-120, Jan. 2015.

[10] R. E. Miles, G. Millington, R. D. Pollard, D. P. Steenson, J. M. Chamberlain, and M. Henini, "An accurate equivalent circuit model of resonant tunnelling diodes" Electron. Lett., vol. 27, no. 5, pp. 427-428, Feb. 1991.

[11] M. E. Hines, "High-frequency negative-resistance circuit principles for Esaki diode applications," Bell Syst. Tech. J., vol. 39, no. 3, pp. 477-513, May 1960.

[12] I. T. Frisch, "A stability criterion for tunnel diodes," Proc. IEEE, vol. 52, no. 8, pp. 922-923, Aug. 1964.

[13] S. D. Stearns, "Non-Foster circuits and stability theory," Proc. IEEE Antennas Propag. Soc. Int. Symp., pp. 1942-1945, Jul. 2011.

[14] S. D. Stearns, "Circuit stability theory for non-Foster Circuits," IEEE MTT-S Int. Microw. Symp. Dig., Seattle, WA, June 2-7, 2013.

[15] M. L. Edwards and J. H. Sinsky, "A new criterion for linear 2-port stability using a single geometrically derived parameter," IEEE Trans. Microw. Theory Tech., vol. 40, no. 12, pp. 2303-2311, Dec. 1992.

[16] K. Z. Rajab, Y. F. Fan and Y. Hao, "Characterization of active metamaterials based on negative impedance converters", J. Opt., vol. 57, April 2012.

[17] S. E. Sussman-Fort and R. M. Rudish, "Non-Foster impedance matching of electrically-small antennas," IEEE Trans. Antennas Propag., vol. 57, no. 8, pp. 2230-2341, Aug. 2009.

[18] B. van der Pol, "A New Transformation in Alternating-Current Theory with an Application to the Theory of Audition," Proc. IRE, vol. 18, no. 2, pp. 221-230, Feb. 1930. 